\title{
Data-Driven Smooth Tests When the Hypothesis Is Composite
}

\author{
Wilbert C. M. KALLENBERG and Teresa LEDWINA
}

\begin{abstract}
In recent years several authors have recommended smooth tests for testing goodness of fit. However, the number of components in the smooth test statistic should be chosen well; otherwise, considerable loss of power may occur. Schwarz's selection rule provides one such good choice. Earlier results on simple null hypotheses are extended here to composite hypotheses, which tend to be of more practical interest. For general composite hypotheses, consistency of the data-driven smooth tests holds at essentially any alternative. Monte Carlo experiments on testing exponentiality and normality show that the data-driven version of Neyman's test compares well to other, even specialized, tests over a wide range of alternatives.
\end{abstract}

KEY WORDS: Goodness of fit; Monte Carlo study; Neyman's test; Schwarz's BIC criterion.

\section{INTRODUCTION}

In recent years there has been substantial interest in datadriven procedures for testing goodness of fit (see, e.g., Bickel and Ritov 1992; Eubank and Hart 1992; Eubank, Hart, and LaRiccia 1993; Eubank and LaRiccia 1992; Fan 1996; Inglot and Ledwina 1996; Kallenberg and Ledwina 1994, 1995a,b; Ledwina 1994). Although smooth tests are often recommended for this purpose (see,e.e.g., Milbrodt and Strasser 1990; Rayner and Best 1990), a wrong choice for the number $k$ of components in the test statistic can result in a considerable loss of power. Therefore, a good procedure is needed for choosing a value for $k$ that can be used in practice. Renewed research in the area of smooth tests shows that a deterministic procedure gives no simple answer (see Inglot, Kallenberg, and Ledwina 1994a). Hence Ledwina (1994) introduced a data-driven version of Neyman's test for testing uniformity. An important role in the data-driven smooth test is played by Schwarz's selection rule. It provides the "right" dimension (or, equivalently, number of components) for the smooth test. The selection rule may be seen as the first step, followed by the finishing touch of applying the smooth test in the selected dimension.

To apply this procedure for testing composite hypotheses (e.g., for testing normality or exponentiality), Schwarz's selection rule must be extended. This is done in Section 2 by inserting an estimator of the parameters involved in the composite null hypothesis.

It turns out that the data-driven smooth test with this selection rule is consistent against essentially any alternative. A modification of the selection rule is also presented, which gives a consistent test but is easier to calculate.

To investigate the behavior of the tests for finite sample sizes, Monte Carlo experiments were conducted for testing exponentiality and normality. The results from these experiments are reported in Sections 4 and 5. We also analyze the power behavior of smooth tests for different fixed val-

Wilbert C. M. Kallenberg is Senior Lecturer, Department of Applied Mathematics, University of Twente, 7500 AE Enschede, The Netherlands. Teresa Ledwina is Professor, Institute of Mathematics, Technical University of Wrocław, 50-370 Wrocław, Poland. The research of Teresa Ledwina was supported by grant KBN 350 044. Programming work has been done by Krzysztof Bogdan under partial support of grant KBN 350044 . The authors thank the editor, the associate editor, and the referees. The presentation benefitted from their comments. ues of the number of components and study the behavior of Schwarz's selection rule under alternatives. The new tests work very well and are even competitive with well-known "special" tests, such as Gini's test for exponentiality and the Shapiro-Wilk's test for normality. A more detailed description of the conclusions is given at the end of Sections 4 and 5.

To apply the test in practice, critical values or $p$ values can be simulated. One can also rely on a simple and accurate approximation of critical values and $p$ values, which is presented in Section 3.

Schwarz's selection rule is not the only solution for choosing the dimension. However, a comparison of selection rules with other penalties in Section 6 gives sufficient support to recommend the use of Schwarz's rule for general applications. The article concludes with a real example in Section 7.

\section{DATA-DRIVEN SMOOTH TESTS}

\subsection{Smooth Tests}

Let $X_{1}, X_{2}, \ldots, X_{n}$ be iid random variables with density $f(x)$. We want to test the null hypothesis

$$
H_{0}: f(x) \in\{f(x ; \boldsymbol{\beta}): \boldsymbol{\beta} \in B\},
$$

where $B \subset \mathbb{R}^{q}$ and $\{f(x ; \boldsymbol{\beta}): \boldsymbol{\beta} \in B\}$ is a given family of densities. Of particular interest are the problems of testing for exponentiality wherein, for $\beta>0$

$$
f(x ; \beta)= \begin{cases}0, & x<0 \\ \beta^{-1} \exp \left(-\beta^{-1} x\right), & x>0,\end{cases}
$$

and testing for normality where $f(x ; \boldsymbol{\beta})=(\sqrt{2 \pi} \sigma)^{-1}$ $\exp \left\{-\frac{1}{2}(x-\mu)^{2} / \sigma^{2}\right\}$, for $\boldsymbol{\beta}=(\mu, \sigma)$ with $\mu \in \mathbb{R}$ and $\sigma>0$.

To test the null hypothesis, a sequence of exponential families is built, which extends from the null model using classes of alternatives that become larger and larger as the dimension of the exponential family grows. Testing $H_{0}$ within this exponential family then leads to a standard testing problem.

(C) 1997 American Statistical Association Journal of the American Statistical Association September 1997, Vol. 92, No. 439, Theory and Methods 
To be more precise, consider an orthonormal system

$$
\phi_{0}, \phi_{1}, \phi_{2}, \ldots
$$

in $L_{2}([0,1])$ with bounded functions $\phi_{1}, \phi_{2}, \ldots$ and $\phi_{0}(x) \equiv$ 1. The functions $\phi_{1}, \phi_{2}, \ldots$ are not necessarily uniformly bounded. Typical examples of $\phi_{0}, \phi_{1}, \ldots, \phi_{k}$ are the orthonormal Legendre polynomials on $[0,1]$, corresponding to Neyman's smooth test and the cosine system, given by $\phi_{j}(x)=\sqrt{2} \cos (j \pi x), j=1,2, \ldots$.

Now let $F(x ; \boldsymbol{\beta})$ be the distribution function of $X_{i}$ when $\boldsymbol{\beta}$ applies. For $k=1,2, \ldots$ define exponential families (with respect to $\theta$ ) by their density

$$
g(x ; \boldsymbol{\theta}, \boldsymbol{\beta})=\exp \left\{\boldsymbol{\theta} \circ \boldsymbol{\phi}[F(x ; \boldsymbol{\beta})]-\psi_{k}(\boldsymbol{\theta})\right\} f(x ; \boldsymbol{\beta}),
$$

where

$$
\begin{aligned}
\boldsymbol{\theta}=\left(\theta_{1}, \ldots, \theta_{k}\right), \quad \boldsymbol{\phi} & =\left(\phi_{1}, \ldots, \phi_{k}\right), \\
& \psi_{k}(\boldsymbol{\theta})=\log \int_{0}^{1} \exp \{\boldsymbol{\theta} \circ \boldsymbol{\phi}(y)\} d y,
\end{aligned}
$$

and $\circ$ stands for the inner product in $\mathbb{R}^{k}$. In what follows, the Euclidean norm in $\mathbb{R}^{k}$ (or $\mathbb{R}^{q}$ ) is denoted by $\|\cdot\|$. When there is no confusion, the dimension $k$ is sometimes suppressed in the notation.

Testing $H_{0}$ within the exponential family (1) means testing $H: \boldsymbol{\theta}=0$ against $\boldsymbol{\theta} \neq 0$. An obvious test statistic for this testing problem is the score statistic (see Javitz 1975; Kopecky and Pierce 1979; Neyman 1980; Rayner and Best 1989; Thomas and Pierce 1979). Denoting by I the $k \times k$ identity matrix and the transpose of a matrix or vector by ', the score statistic is given by

$$
W_{k}=n \mathbf{Y}_{n}^{\prime}(\hat{\boldsymbol{\beta}})\{\mathbf{I}+\mathbf{R}(\hat{\boldsymbol{\beta}})\} \mathbf{Y}_{n}(\hat{\boldsymbol{\beta}}),
$$

where, writing $E_{\boldsymbol{\beta}}$ for the expected value under $f(x ; \boldsymbol{\beta})$,

$$
\begin{array}{r}
\mathbf{Y}_{n}(\boldsymbol{\beta})=\left(\bar{\phi}_{1}(\boldsymbol{\beta}), \ldots, \bar{\phi}_{k}(\boldsymbol{\beta})\right)^{\prime} \\
=n^{-1} \sum_{i=1}^{n}\left(\phi_{1}\left[F\left(X_{i} ; \boldsymbol{\beta}\right)\right], \ldots, \phi_{k}\left[F\left(X_{i} ; \boldsymbol{\beta}\right)\right]\right)^{\prime}, \\
\mathbf{I}_{\boldsymbol{\beta}}=\left\{-E_{\boldsymbol{\beta}} \frac{\partial}{\partial \beta_{t}} \phi_{j}[F(X ; \boldsymbol{\beta})]\right\}_{t=1, \ldots, q, \jmath=1, \ldots, k}, \\
\mathbf{I}_{\boldsymbol{\beta} \boldsymbol{\beta}}=\left\{-E_{\boldsymbol{\beta}} \frac{\partial^{2}}{\partial \beta_{t} \partial \beta_{u}} \log f(X ; \boldsymbol{\beta})\right\}_{t=1, \ldots, q, u=1, \ldots, q} \\
\mathbf{R}(\boldsymbol{\beta})=\mathbf{I}_{\boldsymbol{\beta}}^{\prime}\left(\mathbf{I}_{\boldsymbol{\beta} \boldsymbol{\beta}}-\mathbf{I}_{\boldsymbol{\beta}} \mathbf{I}_{\boldsymbol{\beta}}^{\prime}\right)^{-1} \mathbf{I}_{\boldsymbol{\beta}},
\end{array}
$$

and $\hat{\boldsymbol{\beta}}$ is the maximum likelihood estimator (MLE) of $\boldsymbol{\beta}$ under $H_{0}$.

\subsection{Selection Rules and Data-Driven Smooth Tests}

To choose the "right" dimension, an extension of Schwarz's (1978) Bayesian information criterion (BIC) to the composite hypothesis case is introduced. The likelihood of the independent random variables $X_{1}, \ldots, X_{n}$, each having density (1), is given by

$$
\exp \left\{n\left[\mathbf{Y}_{n}(\boldsymbol{\beta}) \circ \boldsymbol{\theta}-\psi_{k}(\boldsymbol{\theta})\right]\right\} \prod_{i=1}^{n} f\left(X_{i} ; \boldsymbol{\beta}\right) .
$$

In Schwarz's rule the maximized log-likelihood is penalized with $k / 2 \log n$ for dimension $k$. Therefore, when $\boldsymbol{\beta}$ is known, this yields

$$
\begin{aligned}
S(\boldsymbol{\beta})=\min \{k: 1 \leq k \leq d(n), & L_{k}(\boldsymbol{\beta}) \\
& \left.\geq L_{j}(\boldsymbol{\beta}), j=1, \ldots, d(n)\right\}
\end{aligned}
$$

where

$$
L_{k}(\boldsymbol{\beta})=n \sup _{\boldsymbol{\theta} \in \mathbb{R}^{k}}\left\{\boldsymbol{\theta} \circ \mathbf{Y}_{n}(\boldsymbol{\beta})-\psi_{k}(\boldsymbol{\theta})\right\}-\frac{1}{2} k \log n .
$$

The number $d(n)$ is the upper bound of the dimension of the exponential families under consideration. It turns out that $S(\boldsymbol{\beta})$ is stable for large $d(n)$. A more detailed discussion on the choice of $d(n)$ is given in Section 6.2.

The extension to the situation where $\beta$ is an unknown nuisance parameter is obtained by inserting $\hat{\boldsymbol{\beta}}$ in formula (2) to obtain

$$
S=S(\hat{\boldsymbol{\beta}}) .
$$

The maximized log-likelihood (which is in fact the loglikelihood ratio statistic for testing $H: \boldsymbol{\theta}=0$ against $\boldsymbol{\theta} \neq 0$ when $\boldsymbol{\beta}$ is known) is locally equivalent to $\frac{1}{2} n\left\|\mathbf{Y}_{n}(\boldsymbol{\beta})\right\|^{2}$. This leads to the following modification $S 2$ (with 2 from squared norm), which is much easier to calculate:

$$
\begin{aligned}
S 2= & S 2(\hat{\boldsymbol{\beta}})=\min \left\{k: 1 \leq k \leq d(n), n\left\|\mathbf{Y}_{n}(\hat{\boldsymbol{\beta}})\right\|_{(k)}^{2}\right. \\
& \left.-k \log n \geq n\left\|\mathbf{Y}_{n}(\hat{\boldsymbol{\beta}})\right\|_{(j)}^{2}-j \log n, j=1, \ldots, d(n)\right\},
\end{aligned}
$$

where the index of the norm denotes the dimension.

The two data-driven smooth test statistics are now defined by

$$
\begin{gathered}
W_{S}=W_{S(\hat{\boldsymbol{\beta}})} \\
W_{S 2}=W_{S 2(\hat{\boldsymbol{\beta}})} .
\end{gathered}
$$

The null hypothesis is rejected for large values of $W_{S}$ and $W_{S 2}$.

In the case of a location-scale family $\{f(x ; \boldsymbol{\beta}): \boldsymbol{\beta} \in B\}$, the null distributions of $S, S 2, W_{S}$, and $W_{S 2}$ do not depend on $\boldsymbol{\beta}$. Moreover, if the alternative also belongs to a locationscale family, then the distribution of $S, S 2, W_{S}$, and $W_{S 2}$ do not depend on the location-scale parameter of that family. The same remark applies to location families and to scale families.

Theoretical support of the tests $W_{S}$ and $W_{S 2}$ is given by their consistency. Consider an alternative $P$. Under this alternative, $\hat{\boldsymbol{\beta}}$ will as a rule converge to some element of $B$. This element will be called $\boldsymbol{\beta}$. For instance, for testing exponentiality $\hat{\beta}=\bar{X}$, which converges under $P$ to $E_{P} X$; therefore, in that case under the alternative $P, \beta$ will refer to $E_{P} X$. 
Table 1. $5 \%$ and $10 \%$ Critical Values of $W_{S}$ and $W_{S 2}$ for Testing Exponentiality and Normality

\begin{tabular}{|c|c|c|c|c|c|c|c|c|c|c|c|}
\hline \multicolumn{12}{|c|}{ Testing exponentiality } \\
\hline \multicolumn{12}{|c|}{$d(n)$} \\
\hline$\alpha$ & & 1 & 2 & 3 & 4 & 5 & 6 & 7 & 8 & 9 & 10 \\
\hline \multirow[t]{2}{*}{.05} & $w_{S}$ & 3.592 & 4.445 & 4.790 & 4.960 & 5.020 & 5.063 & 5.086 & 5.086 & 5.086 & 5.086 \\
\hline & $w_{S 2}$ & 3.592 & 4.492 & 4.800 & 4.891 & 4.935 & 4.947 & 4.947 & 4.947 & 4.947 & 4.947 \\
\hline \multirow[t]{2}{*}{.10} & $w_{S}$ & 2.562 & 2.783 & 2.886 & 2.937 & 2.955 & 2.959 & 2.967 & 2.967 & 2.967 & 2.967 \\
\hline & $w_{S 2}$ & 2.562 & 2.781 & 2.864 & 2.905 & 2.921 & 2.937 & 2.937 & 2.937 & 2.937 & 2.937 \\
\hline \multicolumn{12}{|c|}{ Testing normality } \\
\hline \multirow[t]{2}{*}{.05} & $W_{S}$ & 3.817 & 3.820 & 3.825 & 3.847 & 3.854 & 3.854 & 3.854 & 3.855 & 3.855 & 3.855 \\
\hline & $W_{S 2}$ & 3.817 & 3.820 & 3.825 & 3.836 & 3.840 & 3.840 & 3.840 & 3.840 & 3.840 & 3.840 \\
\hline \multirow[t]{2}{*}{.10} & $W_{S}$ & 2.725 & 2.726 & 2.728 & 2.745 & 2.750 & 2.757 & 2.757 & 2.757 & 2.757 & 2.757 \\
\hline & $w_{S 2}$ & 2.725 & 2.726 & 2.728 & 2.735 & 2.734 & 2.734 & 2.734 & 2.734 & 2.734 & 2.734 \\
\hline
\end{tabular}

NOTE: Each case is based on 10,000 samples; $n=50$.

We consider alternatives $P$ to the family $\{f(x ; \boldsymbol{\beta}): \boldsymbol{\beta} \in$ $B\}$ for which there exists (for the $\boldsymbol{\beta}$ associated with $\hat{\boldsymbol{\beta}}$ and $P) K(\boldsymbol{\beta})$ such that

$$
\begin{aligned}
E_{P} \phi_{1}(F(X ; \boldsymbol{\beta}))=\cdots= & E_{P} \phi_{K(\boldsymbol{\beta})-1}(F(X ; \boldsymbol{\beta})) \\
& =0, E_{P} \phi_{K(\boldsymbol{\beta})}(F(X, \boldsymbol{\beta})) \neq 0 .
\end{aligned}
$$

Note that if (3) does not hold, then $E_{P} \phi_{j}(F(X ; \boldsymbol{\beta}))=0$ for all $j$, and thus essentially any alternative of interest satisfies (3).

Theorem 2.1. Under the conditions given in the Appendix, the tests based on $W_{S}$ and $W_{S 2}$ are consistent against any alternative satisfying (3).

Consequently, the following corollary.

Corollary 2.1. For testing exponentiality or normality the test based on $W_{S}$ and $W_{S 2}$ with $\left\{\phi_{j}\right\}$, the orthonormal Legendre polynomials on $[0,1]$ are consistent against any alternative $P$ having finite second moment and satisfying (3) if $d(n)=o\left(\{n / \log n\}^{1 / 9}\right)$. The same result holds if $\left\{\phi_{j}\right\}$ is the cosine system, in which case $d(n)=o\left(\{n / \log n\}^{1 / 4}\right)$ suffices for testing exponentiality and for each $\varepsilon>0$, $d(n)=o\left(n^{(1 / 6)-\varepsilon}\right)$ suffices for testing normality.

The proof of Theorem 2.1 was provided by Inglot et al. (1994b). (A more general case is treated in Inglot et al. 1997.) The proof of Corollary 2.1 is provided in the Appendix.

\section{CRITICAL VALUES}

The null hypothesis corresponds to $\boldsymbol{\theta}=0$ in (1) for any dimension. Because the penalty in the selection rules increases substantially with the dimension, it may be expected that under $H_{0}$, the lowest dimension is prevalent. Indeed, it can be shown that the selection rules $S$ and $S 2$ converge under $H_{0}$ in probability to 1 (Inglot et al. 1994b). To show how well this limiting theorem describes the finite-sample

Table 2. Second-Order Approximation of $P\left(W_{S} \leq x\right)$ for Testing Exponentiality With $x$ the 5\% Critical Value From Table 1

\begin{tabular}{crrrrrr}
\hline \hline$x$ & 4.445 & 4.790 & 4.960 & 5.020 & 5.063 & 5.086 \\
\hline Appr. & .942 & .947 & .949 & .950 & .950 & .950 \\
\hline
\end{tabular}

behavior, the null distribution of the selection rules was simulated for testing exponentiality and normality. In all of the simulations we take smooth tests and selection rules, with the $\phi_{j}$ 's being the orthonormal Legendre polynomials on $[0,1]$. The simulations are performed in a similar way to that described by Ledwina (1994). Every Monte Carlo experiment reported here was repeated 10,000 times. Hence the standard deviation of the simulated powers and probabilities concerning the selection rules does not exceed $(40,000)^{-1 / 2}=.005$.

Let $n=50$ and $d(50)=10$. Then for testing exponentiality, we found the empirical relative frequencies of choosing $s=1,2$, and 3 to be $.96, .03$, and .01 . Similarly, for testing normality, these probabilities were $.99, .00$, and .01 .

The same holds for $S 2$. Thus it appears that the selection rules do indeed concentrate on dimension 1 , the concentration in the case of testing normality being even stronger than that for testing exponentiality.

Under classical regularity conditions, we have under $H_{0}$ that

$$
\sqrt{n} \mathbf{Y}_{n}(\hat{\boldsymbol{\beta}}) \stackrel{D}{\rightarrow} N\left(0, \mathbf{I}-\mathbf{A}_{\boldsymbol{\beta}}\right), \quad \text { with } \quad \mathbf{A}_{\boldsymbol{\beta}}=\mathbf{I}_{\boldsymbol{\beta}}^{\prime} \mathbf{I}_{\boldsymbol{\beta} \boldsymbol{\beta}}^{-1} \mathbf{I}_{\boldsymbol{\beta}} .
$$

Because

$$
\begin{aligned}
\mathbf{A}_{\beta} & =\mathbf{I}_{\beta}^{\prime} \mathbf{I}_{\boldsymbol{\beta} \beta}^{-1}\left(\mathbf{I}_{\beta \beta}-\mathbf{I}_{\boldsymbol{\beta}} \mathbf{I}_{\beta}^{\prime}\right)\left(\mathbf{I}_{\beta \beta}-\mathbf{I}_{\beta} \mathbf{I}_{\beta}^{\prime}\right)^{-1} \mathbf{I}_{\beta} \\
& =\mathbf{R}(\boldsymbol{\beta})-\mathbf{A}_{\beta} \mathbf{R}(\boldsymbol{\beta}),
\end{aligned}
$$

we get

$$
\left(\mathbf{I}-\mathbf{A}_{\beta}\right)(\mathbf{I}+\mathbf{R}(\boldsymbol{\beta}))=\mathbf{I}+\mathbf{R}(\boldsymbol{\beta})-\mathbf{A}_{\boldsymbol{\beta}}-\mathbf{A}_{\boldsymbol{\beta}} \mathbf{R}(\boldsymbol{\beta})=\mathbf{I} .
$$

It now follows that under $H_{0}, W_{k} \stackrel{D}{\rightarrow} \chi_{k}^{2}$, where $\chi_{k}^{2}$ denotes a random variable with a chi-squared distribution with $k$ df. Hence, due to the concentration of $S$ and $S 2$ on dimension 1 , we obtain $W_{S} \stackrel{D}{\rightarrow} \chi_{1}^{2}$ and $W_{S 2} \stackrel{D}{\rightarrow} \chi_{1}^{2}$. Therefore, one might expect the critical values to be close to those of a chi-squared random variable with $1 \mathrm{df}$. A summary of some simulated critical values for testing exponentiality and normality with $n=50$ is given in Table 1 .

Although the results are satisfactory for testing normality, for testing exponentiality, substantial differences occur between the simulated and asymptotic critical values. In view of the higher concentration on dimension 1 for $S$ and 
Table 3. Estimated $P(S(\hat{\beta})=s)$ and $P(S 2(\hat{\beta})=s)$ Under Alternatives (in \%) When Testing Exponentiality

\begin{tabular}{|c|c|c|c|c|c|c|c|c|}
\hline \multirow{2}{*}{\multicolumn{2}{|c|}{ Alternative }} & \multicolumn{6}{|c|}{$s$} & \multirow[b]{2}{*}{$7-10$} \\
\hline & & 1 & 2 & 3 & 4 & 5 & 6 & \\
\hline \multirow[t]{2}{*}{$e 1$} & $P(S(\hat{\beta})=s)$ & 56 & 40 & 2 & 2 & & & \\
\hline & $P(S 2(\hat{\beta})=s)$ & 62 & 36 & 2 & & & & \\
\hline \multirow[t]{2}{*}{$e 2$} & $P(S(\hat{\beta})=s)$ & 58 & 14 & 24 & 2 & 2 & & \\
\hline & $P(S 2(\hat{\beta})=S)$ & 64 & 16 & 19 & 1 & & & \\
\hline \multirow[t]{2}{*}{$e 3$} & $P(S(\hat{\beta})=s)$ & 69 & 26 & 2 & 2 & & & \\
\hline & $P(S 2(\hat{\beta})=s)$ & 64 & 27 & 2 & 4 & 1 & 1 & 2 \\
\hline \multirow[t]{2}{*}{$e 4$} & $P(S(\hat{\beta})=s)$ & 16 & 72 & 6 & 5 & 1 & 1 & \\
\hline & $P(S 2(\hat{\beta})=S)$ & 22 & 64 & 14 & 1 & & & \\
\hline \multirow[t]{2}{*}{$e 5$} & $P(S(\hat{\beta})=s)$ & 27 & 41 & 14 & 12 & 3 & 2 & 1 \\
\hline & $P(S 2(\hat{\beta})=s)$ & 43 & 49 & 7 & 1 & & & \\
\hline \multirow[t]{2}{*}{$e 6$} & $P(S(\hat{\beta})=s)$ & 71 & 18 & 7 & 3 & 1 & & 1 \\
\hline & $P(S 2(\hat{\beta})=s)$ & 62 & 27 & 4 & 3 & 1 & 1 & 3 \\
\hline
\end{tabular}

NOTE: Each case is based on 10,000 samples; $n=50, d(50)=10$.

$S 2$ in the case of testing normality, this is not surprising. Although in the case of testing exponentiality, $S$ and $S 2$ also tend to concentrate on dimension 1 , there is still a difference, because as a rule when $S$ or $S 2$ is large, this implies a large value of $W_{S}$ or $W_{S 2}$ as well.

To get $p$ values (or critical values), one may perform simulations. However, it is also possible to apply an approximation, which is much more accurate than the chi-squared approximation but is still simple. We may write

$$
\begin{aligned}
P\left(W_{S} \leq x\right)= & P\left(W_{1} \leq x, S=1\right) \\
& +P\left(W_{2} \leq x, S=2\right)+P\left(W_{S} \leq x, S \geq 3\right) .
\end{aligned}
$$

Under $H_{0}, P(S \geq 3)$ is negligible. The event $\{S=1\}$ means that dimension 1 "beats" dimension 2,3 , and so on. The most important event is that dimension 1 "beats" dimension 2, and this is approximately equal to $\left\{n\left(\bar{\phi}_{2}(\hat{\boldsymbol{\beta}})\right)^{2} \leq\right.$ $\log n\}$. Similarly, for $\{S=2\}$, the most important part corresponds to dimension 2 "beating" dimension 1 . Therefore, under $H_{0}$, we get the following approximation:

$$
\begin{aligned}
P\left(W_{S} \leq x\right) \approx P\left(W_{1} \leq\right. & \left.x, n\left(\bar{\phi}_{2}(\hat{\boldsymbol{\beta}})\right)^{2} \leq \log n\right) \\
& +P\left(W_{2} \leq x, n\left(\bar{\phi}_{2}(\hat{\boldsymbol{\beta}})\right)^{2}>\log n\right) .
\end{aligned}
$$

Except for very large $x, n\left(\bar{\phi}_{2}(\hat{\boldsymbol{\beta}})\right)^{2}>\log n$ implies $W_{2}>x$, and hence we omit the second term in the approximation. Taking the limit distribution of $\sqrt{n}\left(\bar{\phi}_{1}(\hat{\boldsymbol{\beta}}), \bar{\phi}_{2}(\hat{\boldsymbol{\beta}})\right)$, writing $\mathbf{A}$ for $\mathbf{A}_{\hat{\boldsymbol{\beta}}}$ (note that for location/scale families $\mathbf{A}$ does not depend on $\boldsymbol{\beta}$ ), and defining $a, b, c$, and $\rho$ by

$$
\mathbf{I}-\mathbf{A}=\left(\begin{array}{cc}
a & b \\
b & c
\end{array}\right), \quad \rho=b / \sqrt{a c}
$$

we get as an approximation [Bickel and Doksum 1977, (1.4.18), p. 25]

$$
P\left(W_{S} \leq x\right) \approx P\left(\left\{\sqrt{1-\rho^{2}} U_{1}+\rho U_{2}\right\}^{2} \leq x, c U_{2}^{2} \leq \log n\right),
$$

where $U_{1}$ and $U_{2}$ are independent and $N(0,1)$ distributed.

A further look at the integration region, noting that a $N(0,1)$-distributed random variable has almost no probability mass above $2 \log n$, suggests the following very simple approximation:

$$
\begin{aligned}
P\left(W_{S} \leq x\right) & \approx 2 \Phi(\sqrt{x})-1 \\
& -2\left[1-\Phi\left\{\left(c^{-1} \log n\right)^{1 / 2}\right\}\right][\Phi(b(x))-\Phi(a(x))],
\end{aligned}
$$

with

$$
\begin{gathered}
b(x)=\left[\sqrt{x}-\rho \frac{1}{2}\left\{\sqrt{\frac{\log n}{c}}+\sqrt{2 \log n}\right\}\right] / \sqrt{1-\rho^{2}}, \\
a(x)=\left[-\sqrt{x}-\rho \frac{1}{2}\left\{\sqrt{\frac{\log n}{c}}+\sqrt{2 \log n}\right\}\right] / \sqrt{1-\rho^{2}},
\end{gathered}
$$

and $\Phi$ denoting the standard normal distribution function. In case of the exponential distribution we get $c=31 / 36$ and $\rho=-\sqrt{15 / 31}$. Inserting for $x$ the simulated critical values of Table 1 (with $d(n) \geq 2$ ), we get the results shown in Table 2. As seen in Table 2, the approximation appears to work quite well in the exponential case. For testing normality, we get $\rho=0$ and $c=1-15 /\left(2 \pi^{2}\right)=.2401$. For instance, if $n=50$, then $\left(c^{-1} \log n\right)^{1 / 2}=4.0366$, and hence $P\left(c U_{2}^{2} \geq \log n\right)=2\{1-\Phi(4.0366)\}=.00005$, implying that the approximation above is very close to the chi-squared-one approximation. This explains why in the normal case, the simulated critical values are close to the chi-squared-one critical values (cf. Table 1).

Both examples (testing exponentiality and normality) show that the foregoing approximation gives $p$ values in a simple and accurate way and can also serve to find critical values. The approximation can be used for $W_{S 2}$ as well.

\section{POWER FOR TESTING EXPONENTIALITY}

Let $X_{1}, X_{2}, \ldots$ be iid random variables each distributed according to some probability distribution $P$. For testing exponentiality, $\hat{\beta}=\bar{X}$, which converges under $P$ to $E_{P} X$ and thus, under alternatives, $\beta=E_{P} X$. We consider $P$ to be an alternative if there exists $K(\beta)=K\left(E_{P} X\right)$ such that

$$
\begin{aligned}
E_{P} \phi_{1}(F(X ; \beta))=\cdots & =E_{P} \phi_{K(\beta)-1}(F(X ; \beta)) \\
& =0, E_{P} \phi_{K(\beta)}(F(X ; \beta)) \neq 0,
\end{aligned}
$$

with $F(x ; \beta)=1-\exp \left(-\beta^{-1} x\right)$, the distribution function of the exponential distribution.

While under $H_{0}$ the selection rules concentrate on dimension 1 , under alternatives we have

$$
\lim _{n \rightarrow \infty} P(S(\hat{\beta}) \geq K(\beta))=1
$$

and

$$
\lim _{n \rightarrow \infty} P(S 2(\hat{\beta}) \geq K(\beta))=1
$$

(see Inglot et al. 1994b). To illustrate this result, we present some Monte Carlo results. For testing exponentiality, we present the following alternatives: 

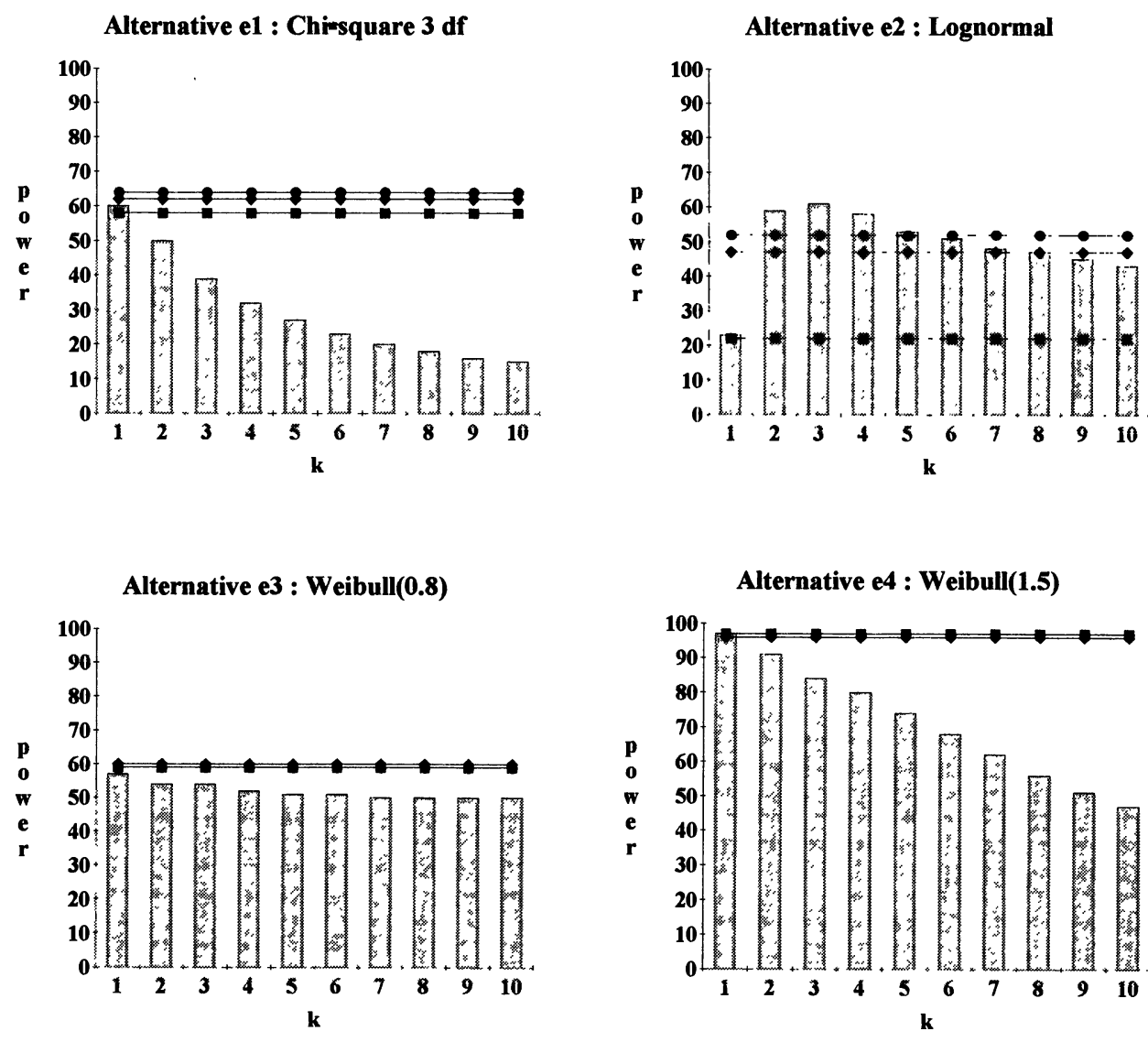

power $\mathbf{W}_{\mathbf{S}}=$ power $\mathbf{G}$

power $W_{S}=$ power $G$
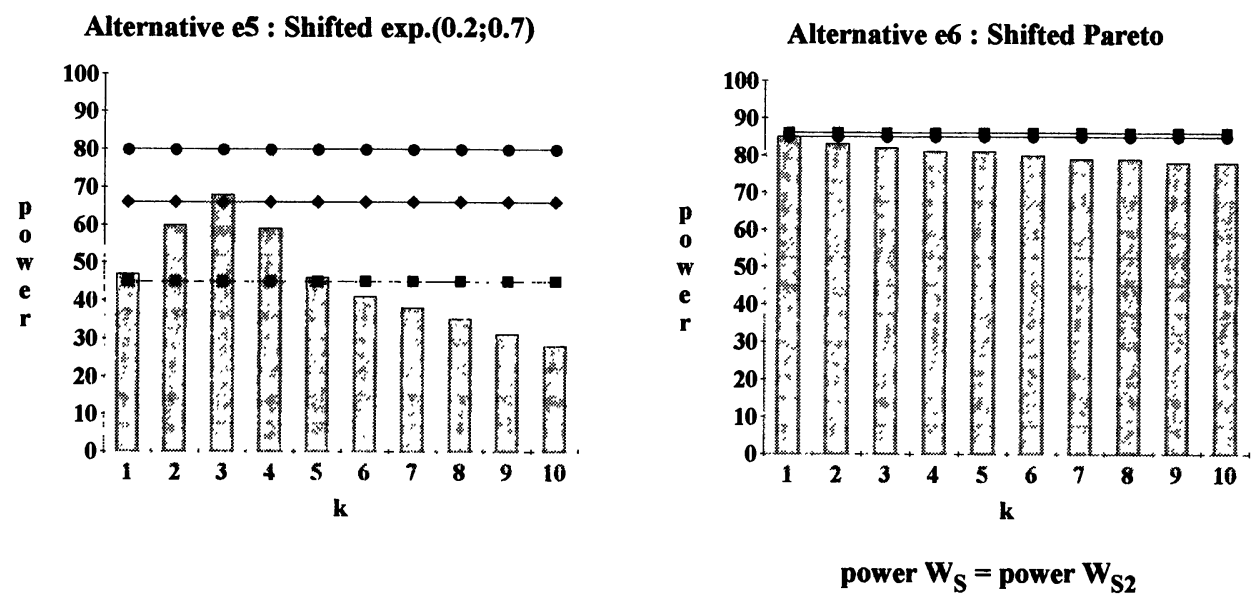

Figure 1. Estimated Powers (in \%) of $W_{k}$ (bars), $W_{S}(-\bullet-), W_{S 2}(-\bullet), G(-\square-)$ Testing Exponentiality; $\alpha=.10, n=50 ; d(50)=$ 10. Each case is based on 10,000 samples.

\begin{tabular}{clll} 
Notation & \multicolumn{1}{c}{ Alternative } & \multicolumn{2}{c}{ Density } \\
\hline$e 1$ & $\chi_{3}^{2}$ & $\left\{2^{3 / 2} \Gamma\left(\frac{3}{2}\right)\right\}^{-1} x^{1 / 2} \exp \left(-\frac{1}{2} x\right)$, & $x>0$ \\
$e 2$ & Lognormal & $(x \sqrt{2 \pi})^{-1} \exp \left\{-\frac{1}{2}(\log x)^{2}\right\}$, & $x>0$ \\
$e 3$ & Weibull (.8) & $.8 x^{-.2} \exp \left(-x^{.8}\right)$, & $x>0$ \\
$e 4$ & Weibull (1.5) & $\frac{3}{2} x^{1 / 2} \exp \left(-x^{3 / 2}\right)$, & $x>0$ \\
$e 5$ & Shifted exponen- & $.7 \exp \{-(x-.2) .7\}$, & $x>.2$ \\
& $\quad$ tial (.2;.7) & & $x>0$
\end{tabular}

Table 3 shows the behavior of the selection rules under alternatives when testing exponentiality against these alternatives.

It is seen that the behavior under alternatives may be quite different from that under $H_{0}$ (see Sec. 3). Indeed, according to the theory, there is less concentration on dimension 1 , and the theoretical similarity between $S(\hat{\beta})$ and $S 2(\hat{\beta})$ is reflected in the simulation results.

Theoretical support of the tests $W_{S}$ and $W_{S 2}$ is given by their consistency; see Theorem 2.1 and Corollary 2.1.

For power comparison in the finite-sample case, we consider $W_{k}(k=1, \ldots, 10), W_{S}, W_{S 2}$, and $G$, Gini's test. This last test is called "powerful against a variety of alternatives" by Gail and Gastwirth (1978) and turned out to perform well in the study of Ascher (1990). It is also used for the sake of comparison by Rayner and Best (1989, p. 88) and 
Table 4. Estimated $P(S(\hat{\boldsymbol{\beta}})=s)$ and $P(S 2(\hat{\boldsymbol{\beta}})=s)$ Under Alternatives (in \%) When Testing Normality

\begin{tabular}{|c|c|c|c|c|c|c|c|c|c|c|c|}
\hline & \multirow[b]{2}{*}{ Alternative } & \multicolumn{10}{|c|}{$s$} \\
\hline & & 1 & 2 & 3 & 4 & 5 & 6 & 7 & 8 & 9 & 10 \\
\hline \multirow[t]{2}{*}{$n 1$} & $P(S(\hat{\beta})=s)$ & 30 & & & 3 & 1 & 58 & 6 & 2 & & \\
\hline & $P(S 2(\hat{\boldsymbol{\beta}})=s)$ & 40 & & & 4 & 1 & 33 & 18 & 4 & & 1 \\
\hline \multirow[t]{2}{*}{$n 2$} & $P(S(\hat{\beta})=s)$ & 97 & 1 & 1 & 1 & & & & & & \\
\hline & $P(S 2(\hat{\beta})=s)$ & 97 & 1 & 1 & 1 & & & & & & \\
\hline \multirow[t]{2}{*}{$n 3$} & $P(S(\hat{\boldsymbol{\beta}})=s)$ & 79 & & 14 & 2 & 3 & & & & & \\
\hline & $P(S 2(\hat{\boldsymbol{\beta}})=s)$ & 82 & & 14 & 2 & 1 & & & & & \\
\hline \multirow[t]{2}{*}{$n 4$} & $P(S(\hat{\beta})=S)$ & 45 & & 44 & 7 & 1 & 1 & & 1 & & \\
\hline & $P(S 2(\hat{\boldsymbol{\beta}})=s)$ & 47 & & 47 & 6 & 1 & & & & & \\
\hline \multirow[t]{2}{*}{$n 5$} & $P(S(\hat{\beta})=S)$ & 87 & 2 & 9 & 1 & 1 & & & & & \\
\hline & $P(S 2(\beta)=S)$ & 88 & 2 & 9 & 1 & & & & & & \\
\hline \multirow[t]{2}{*}{$n 6$} & $P(S(\hat{\beta})=S)$ & 74 & & 19 & 3 & 4 & 1 & & & & \\
\hline & $P(S 2(\hat{\beta})=s)$ & 76 & & 21 & 2 & 1 & & & & & \\
\hline
\end{tabular}

NOTE: Each case is based on 10,000 samples; $n=50 ; d(50)=10$.

by LaRiccia (1991).

The conclusions from the simulation results presented in Figure 1 and from other simulation results that we have performed (see, e.g., Kallenberg and Ledwina 1994) are as follows:

1. A wrong choice of the number $k$ of components in $W_{k}$ may give a considerable loss of power.

2. The power of $W_{S}$ and $W_{S 2}$ is high and stable, often as high or even higher than the "best" of $W_{k}$.

3. The tests $W_{S}$ and $W_{S 2}$, although based on general ideas, can compare favorably even with "special" tests for exponentiality, like Gini's test. $W_{S}$ and $W_{S 2}$ often have higher power than Gini's test and in many cases much higher.

4. In most cases, $W_{S}$ and $W_{S 2}$ have similar powers.

\section{POWER FOR TESTING NORMALITY}

Again we consider alternatives of the form (4), now with $\boldsymbol{\beta}=\left(E_{P} X,\left\{\operatorname{var}_{P} X\right\}^{1 / 2}\right)$, corresponding to the MLE $\hat{\boldsymbol{\beta}}$ under normality and with $F(x ; \boldsymbol{\beta})=\Phi(x$; $\boldsymbol{\beta})=\Phi\left(\left(x-E_{P} X\right) /\left\{\operatorname{var}_{P} X\right\}^{1 / 2}\right)$. The same theoretical results as for testing exponentiality apply here.

We consider the following alternatives. Here $Z$ denotes a $N(0,1)$ random variable and $\varphi$ denotes its density.

\begin{tabular}{|c|c|c|}
\hline Notation & Alternative & Density/Definition \\
\hline$n 1$ & Normal mixture & $\begin{array}{l}.33\{2 \varphi(2 x)\}+.33\{2 \varphi(2[x-2])\} \\
\quad+.34\{2 \varphi(2[x-4])\}, \quad-\infty<x<\infty\end{array}$ \\
\hline$n 2$ & Logistic & $e^{x}\left(1+e^{x}\right)^{-2}$ \\
\hline$n 3$ & $L C(0.1 ; 3)$ & $.1 \varphi(x-3)+.9 \varphi(x), \quad-\infty<x<\infty$ \\
\hline$n 4$ & $\begin{array}{l}.65 \text { uniform } \\
\quad+.35 \text { beta }(10,20)\end{array}$ & $\begin{array}{r}.65+.35 x^{9}(1-x)^{19}\{B(10,20)\}^{-1} \\
0<x<1\end{array}$ \\
\hline$n 5$ & $S U(-1 ; 2)$ & $\begin{aligned} Z=-1+2 \sinh ^{-1}(X) & \\
& -\infty<X<\infty\end{aligned}$ \\
\hline$n 6$ & $S B(1.580,1.357)$ & $\begin{array}{r}Z=1.580+1.357 \log \{X(1-X)\} \\
0<X<1\end{array}$ \\
\hline
\end{tabular}

Note that $S B$ is the alternative from the Johnson system with skewness $\sqrt{b_{1}}=.8$ and kurtosis $b_{2}=3.4$.
Table 4 shows the behavior of the selection rules under alternatives when testing normality. Again the behavior under alternatives is different from that under $H_{0}$ (see Sec. 3 ) with less concentration on dimension 1 . The similarity between $S(\hat{\boldsymbol{\beta}})$ and $S 2(\hat{\boldsymbol{\beta}})$ is also clearly exhibited.

For power comparison in the finite-sample case, we use the prominent and often recommended Shapiro-Wilk's test. The corresponding test statistic is denoted by $W$.

The conclusions from the simulation results presented in Figure 2 and from other simulation results that we have performed (see, e.g., Kallenberg and Ledwina 1994) are as follows:

1. A wrong choice of the number $k$ of components in $W_{k}$ may give a considerable loss of power.

2. For skewed alternatives, the power of $W_{S}$ and $W_{S 2}$ is high and stable, as a rule as high as the "best" of $W_{k}$.

3. The tests $W_{S}$ and $W_{S 2}$, although based on general ideas, can compare to skewed alternatives even with "special" tests for normality, like the Shapiro-Wilk's test, and can dominate moment-based tests.

4. The tests $W_{S}$ and $W_{S 2}$ perform reasonably well in the symmetrical case. They compare well both to symmetrical and skewed alternatives to classical tests (such as Anderson-Darling, Cramér-von Mises, Kolmogorov, and Watson) and some new tests such as those given by Gan and Koehler (1990). It is not a big surprise that $W_{S}$ and $W_{S 2}$ perform better for skewed alternatives than for symmetrical alternatives. It is well known that $W_{1}$ provides little or no protection against nearby symmetric alternatives (see Kopecky and Pierce 1979, p. 397). This is easily understood by noting that $E_{P} \phi_{1}\{\Phi(X ; \boldsymbol{\beta})\}=0$ for symmetric alternatives $P$.

5. In most cases $W_{S}$ and $W_{S 2}$ have comparable empirical powers.

In view of the consistency and the simulation results, we feel that the conclusion of Rayner and Best $(1990$, p. 9) "don't use those other methods-use a smooth test!"-may be slightly sharpened to "use a data-driven smooth test."

\section{MODIFICATIONS}

\subsection{Changing the Penalty}

In the selection rules, the penalty $k / 2 \log n$ is used for dimension $k$. An obvious question is what happens for other penalty functions. The theoretical results, briefly described here, can be extended to other penalties as well. It should be noted that different penalties have an effect on $p$ values and critical values. One should either simulate them or adapt the approximation of Section 3 to the situation at hand.

Of course, taking Schwarz's BIC-rule is not the only solution for defining a selection rule. By taking smaller weights, the power will be smaller for smooth and larger for highly oscillating alternatives. For illustration, we consider for $n=$ 50 again the alternatives $e 1, \ldots, e 6$ when testing exponentiality and $n 1, \ldots, n 6$ when testing normality (Fig. 3). The different penalties are $(k / 2) \log \log n=1.4(k / 2)$ (Hannan and Quin 1979), 2.5(k/2), $(k / 2) \log n=3.9(k / 2)$ (which is Schwarz's rule), and $k / 2(2 \log n)=7.8(k / 2)$ (Haughton, 

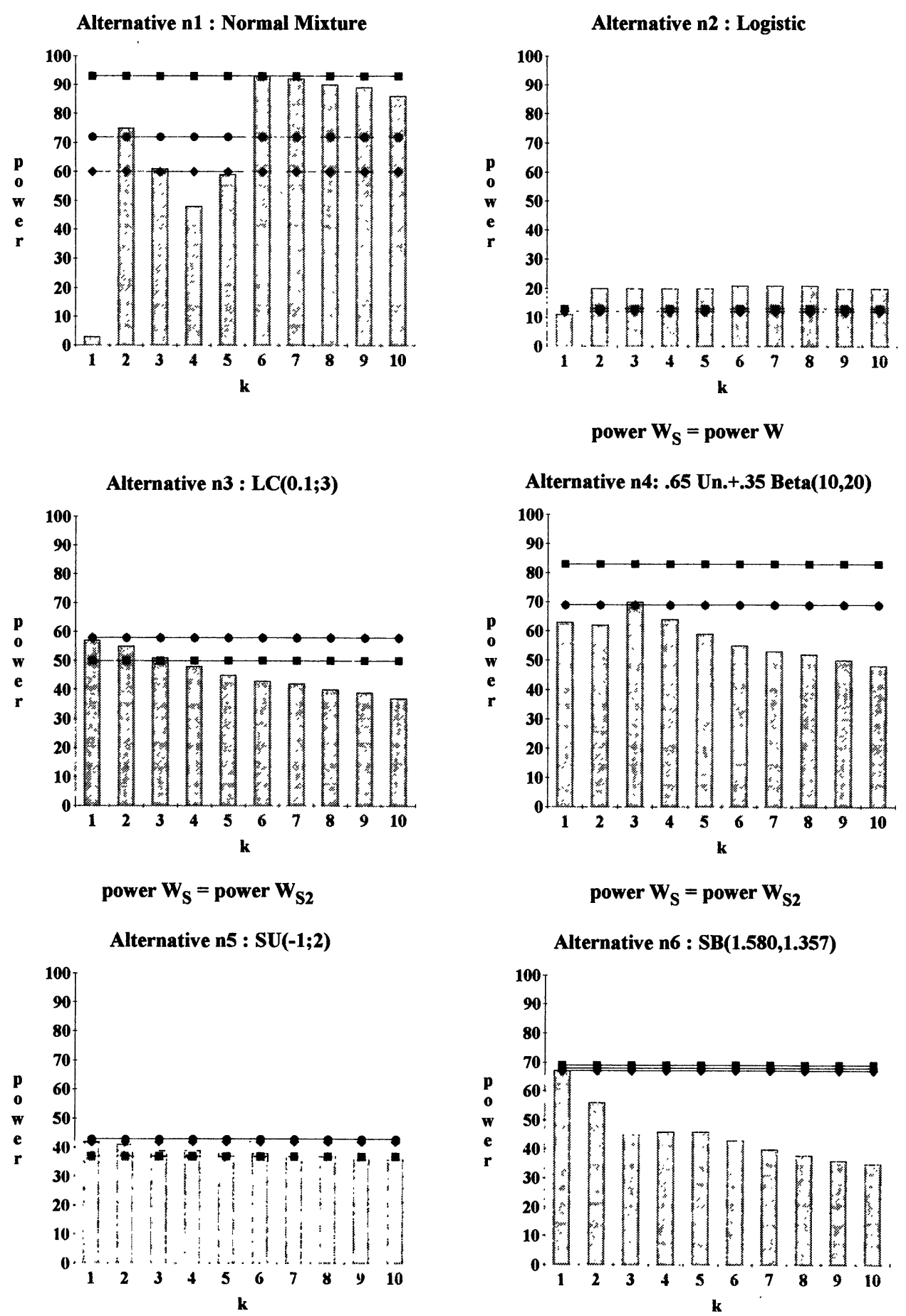

Figure 2. Estimated Powers (in \%) of $W_{k}$ (bar), $W_{S}(-\bullet-), W_{S 2}(-\downarrow), W(-\square-)$ Testing Normality; $\alpha=.05, n=50 ; d(50)=10$. Each case is based on 10,000 samples.

Haughton, and Izenman 1990). For every choice, corresponding critical values are simulated. The selection rule with penalty $c$ instead of $\log n$ is called $S c$.

We conclude from these and other simulations we have performed, that, although other penalties can be considered, there is sufficient support to adopt the Schwarz rule in general.

\subsection{Choice of $d(n)$}

One may ask whether the problem of choosing the number of components $k$ is replaced by the choice of $d(n)$. Such a situation, where the power is not stable as a function of $d(n)$, happens to some order selection criteria (Bogdan 1995). This is certainly not the case if Schwarz's rule is used. In contrast to the power of $W_{k}$ (see Figs. 1 and 2), the power of $W_{S}$ does not change for larger $d(n)$. This is clearly illustrated in Figure 4.

When using $W_{S}$, to ensure convergence of the iterative procedure searching for the MLEs in the exponential family, we recommend $d(n)=6,7$, and 10 for $n=20,30$ and 50, respectively. (For details, see section 3.1 in Ledwina 1994.) When using $W_{S 2}$, no restrictions on $d(n)$ are needed. However, in view of the stability of the power, there is no need to take very large $d(n)$. 


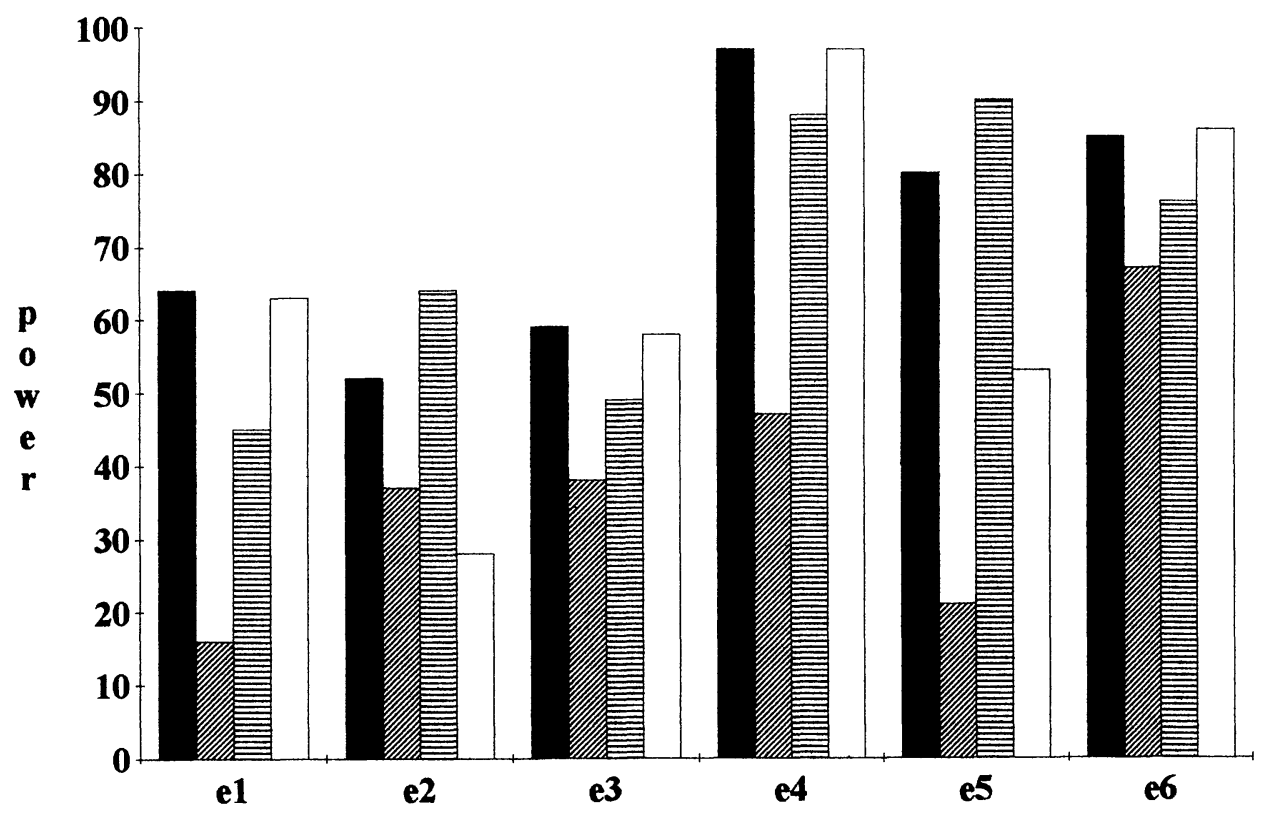

(a)

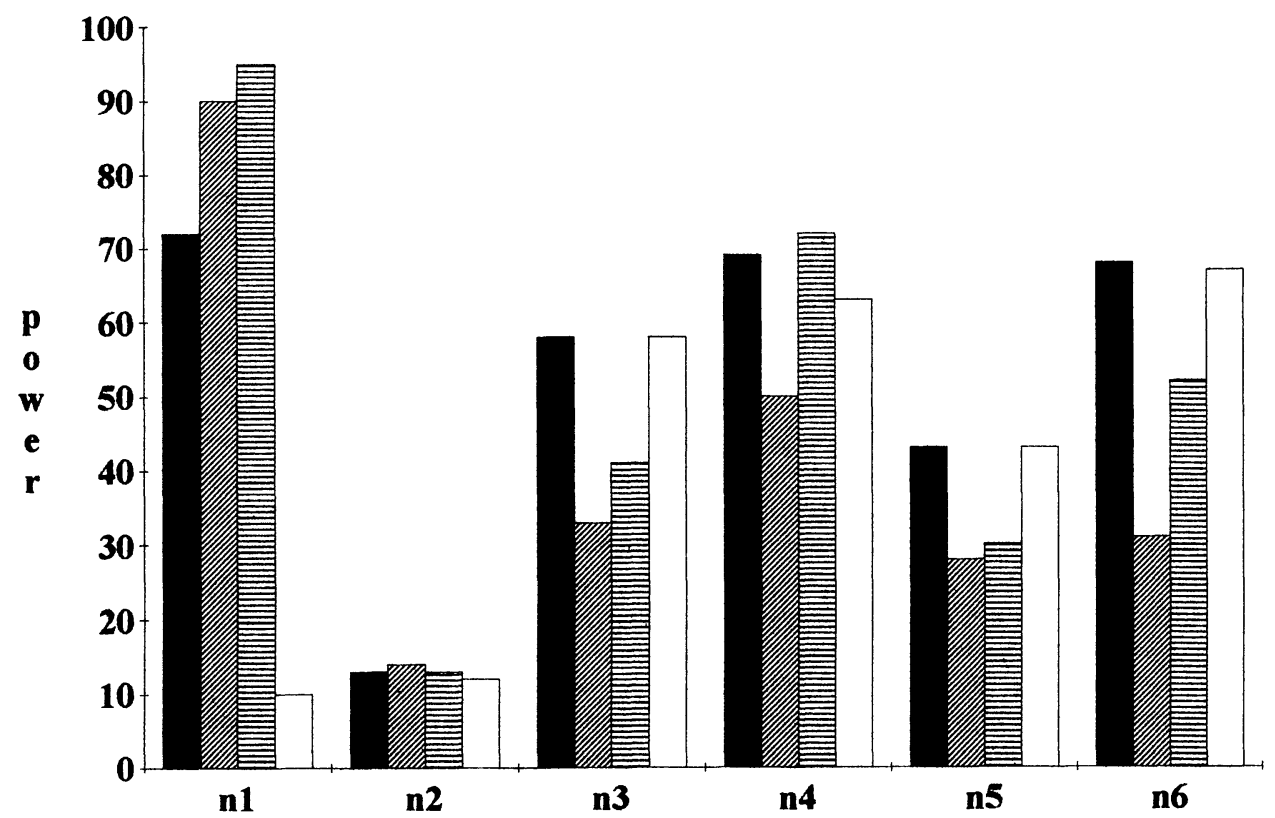

(b)

Figure 3. Estimated Powers (in \%) of $W_{S c}$ for Different Penalties c; (a) for Testing Exponentiality $\alpha=.10, n=50$; (b) for Testing Normality $\alpha=.05, n=50 ; d(50)=10$. Each case is based on 10,000 samples. $\mathbf{\square}, c=\log (n)=3.9 ;$ 还, $c=\log \log (n)=1.4$; 目, $c=2.5 ; \square, c=2$ $\log (n)=7.8$.

\subsection{Starting with Dimension 2}

When testing normality against symmetrical alternatives, the first component $\bar{\phi}_{1}(\hat{\boldsymbol{\beta}})$ will be close to 0 , because $E_{P} \phi_{1}\left(\Phi\left(X_{i} ; \boldsymbol{\beta}\right)\right)=0$. Therefore, one might suggest starting the selection with dimension 2 in that case. Indeed, for symmetric alternatives a higher power is obtained, but for skew alternatives some power is lost.

For $n=50$ it turns out that $W_{S}$ with $S$ starting from 2, is slightly better than taking the penalty $c$ equal to 2.5 .

\section{A NUMERICAL EXAMPLE}

Thomas and Pierce (1979, sec. 5) and Rayner and Best $(1989$, pp. 15,16$)$ discussed a survey in which polychlorinated biphenyl (PCB) concentrations for 65 anacapa birds were tested for normality. (The data are from Risebrough 1972.) The values of $W_{1}, \ldots, W_{10}$ are $4.09,8.19,8.60$, $8.80,9.26,12.65,14.01,14.35,16.00$, and 16.13. Taking $d(65)=10$, the dimension chosen by Schwarz's rule equals 1 , and hence $W_{S}$ equals 4.09 . The $p$ value of $W_{S}$ equals .04 . The same results are obtained for $W_{S 2}$. 


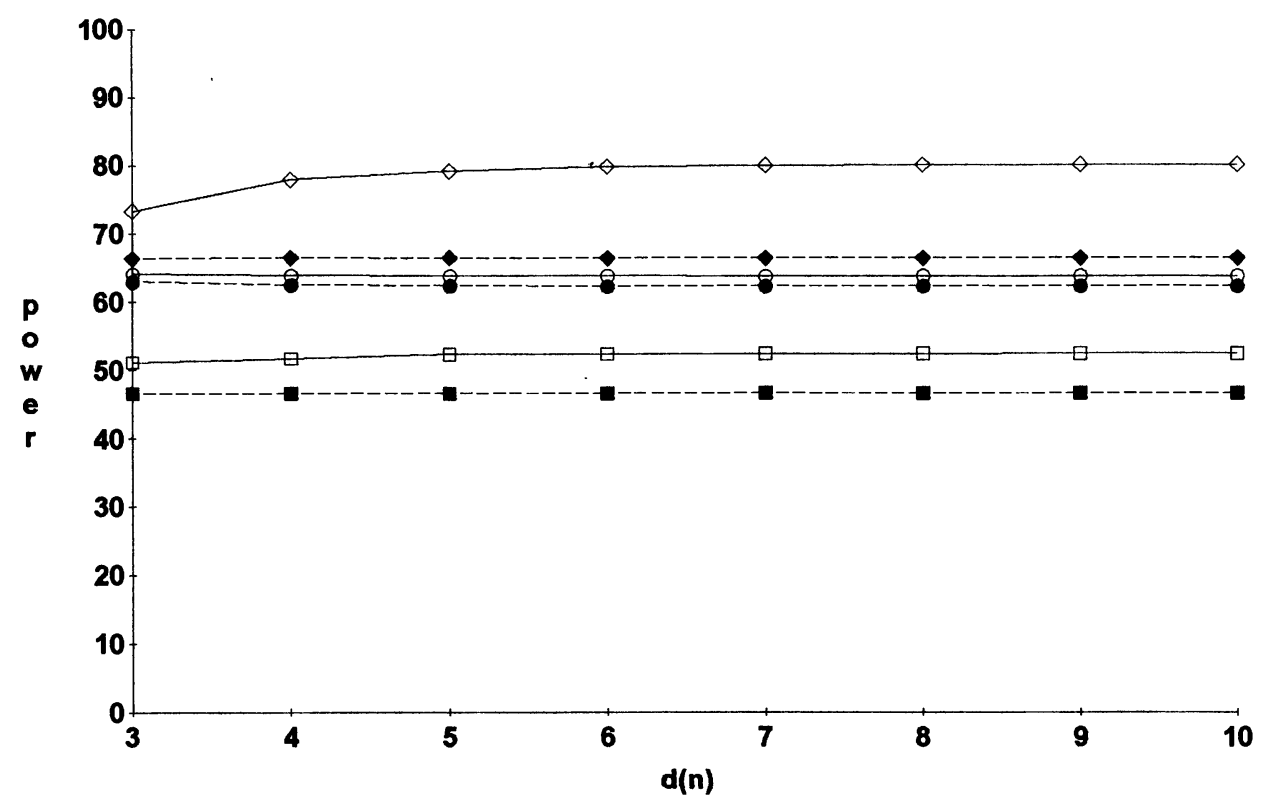

(a)

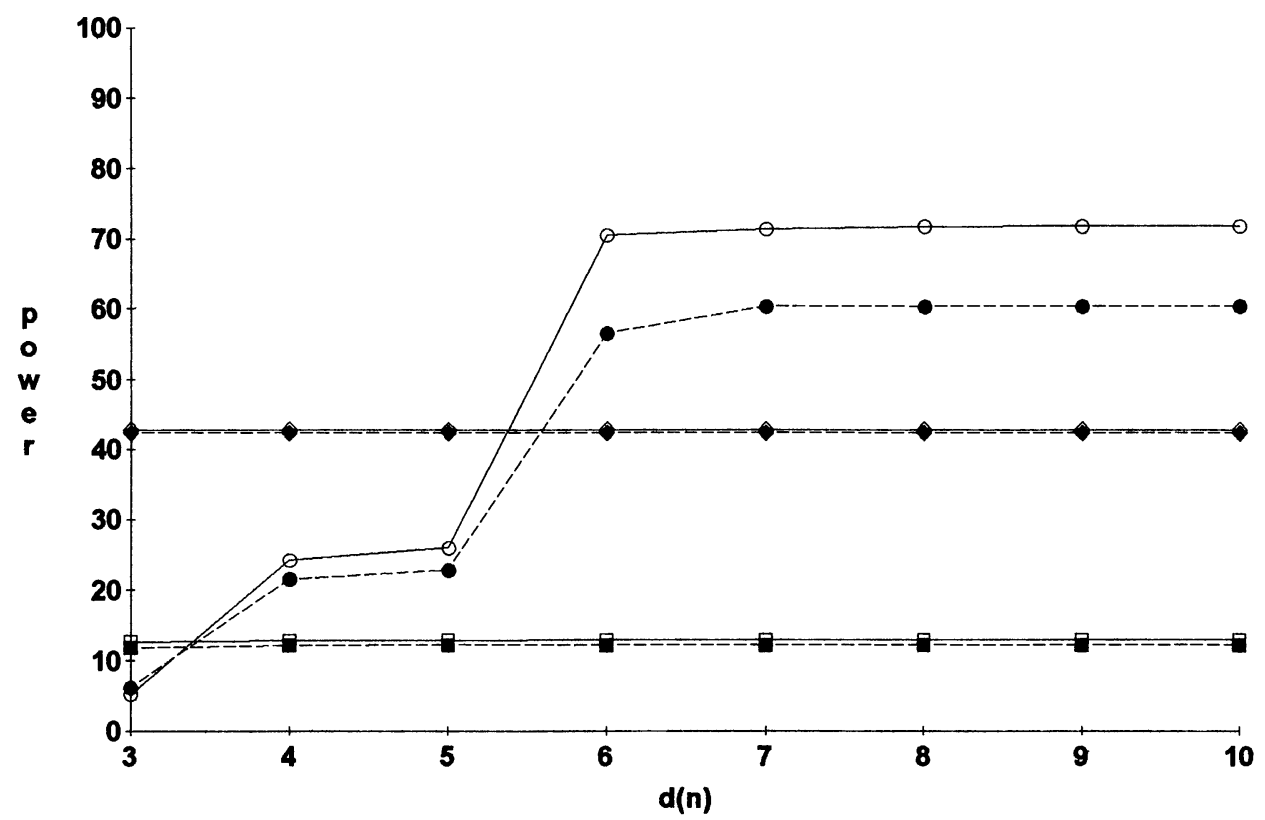

(b)

Figure 4. Estimated Powers (in \%) of $W_{S}$ and $W_{S 2}$ for $d(n)=3 \ldots$. 10; Each Case is Based on 10,000 Samples; - - , $W_{S} ;---, W_{S 2}$. (a) For testing exponentiality $\alpha=.10, n=50 ;-\circ-,-\bullet-, \chi_{3}^{2} ;-\square-,----$, Log normal; $-\diamond-,--\downarrow-$, shifted exponential. (b) For testing

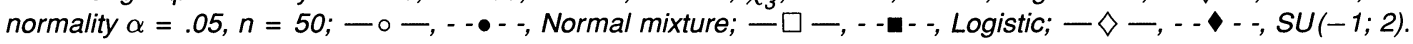

Thomas and Pierce (1979, sec. 5) gave simply $W_{1}, \ldots, W_{4}$ without making a choice, although in general they preferred $W_{2}$. The $p$ value for $W_{2}$ is .017. Further, they present a histogram with an arbitrarily selected number of equal-width classes.

Rayner and Best (1989, p. 15) used the score test (related to some exponential family) with four nonzero components according to their recommendation on p. 84. The resulting $p$ value was about .05 . To give information about the "distance" from normality, they proposed using the GramCharlier type A density estimate based on the four com- ponents (see also our Fig. 5). Moreover, they summarized the data by presenting also a histogram with equal width classes, where the number of classes is arbitrarily chosen, but differs from the one of Thomas and Pierce (1979). In comparison to the work of Thomas and Pierce (1979) and Rayner and Best (1989), we are using the same score test as Thomas and Pierce but with the number of components chosen by Schwarz's rule. We then summarize the data in Figure 5 by providing a likely model (among $d(65)=10$ possible models given by (1)) fitted to the data in the dimension given by Schwarz's rule. 


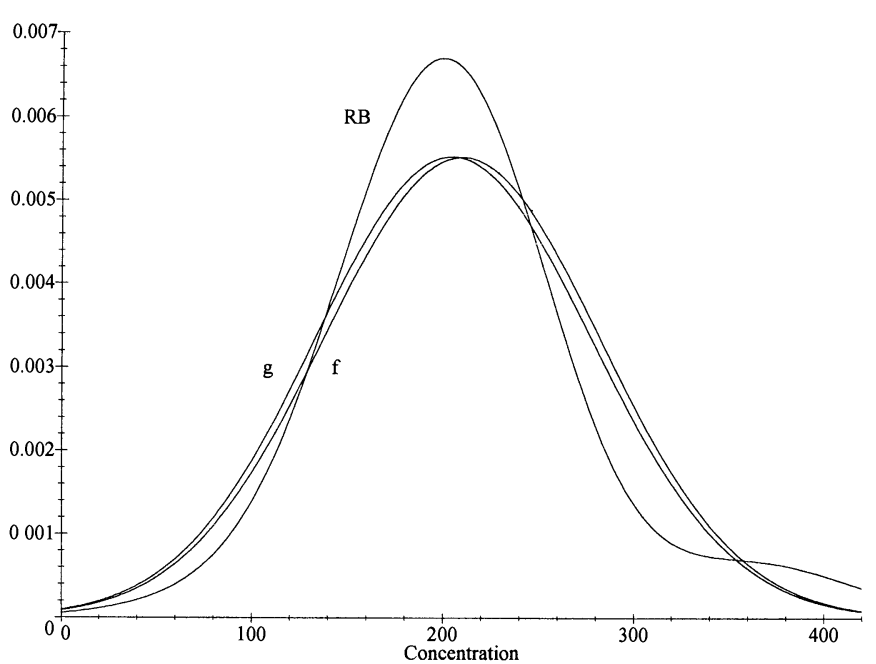

Fig. 5. Density Estimates for PCB Data; Estimated Null Density (f), Estimated Alternative Density (1) (g) and Estimated Gram-Charlier Type $A$ density of Rayner and Best (RB).

\section{APPENDIX: CONDITIONS AND PROOF}

\section{Conditions of Theorem 2.1}

The conditions are divided in several parts: regularity conditions for the null family, conditions concerning the orthonormal system, and conditions on the upper bound $d(n)$ for the dimension of the exponential family.

Denote by $P_{\boldsymbol{\beta}}$ that $X_{\imath}$ density $f(x ; \boldsymbol{\beta})$ and by $E_{\boldsymbol{\beta}}$ and $\operatorname{var}_{\boldsymbol{\beta}}$ the corresponding expected value and variance. For the family $\{f(x ; \boldsymbol{\beta}): \boldsymbol{\beta} \in B\}$ and the alternative $P$, we set the following regularity conditions. The conditions $(\mathrm{r} 1)-(\mathrm{r} 4)$ should hold on an open subset $B_{0}$ of $B$. (The "true" value of $\boldsymbol{\beta}$ under $H_{0}$ is supposed to lie in $B_{0}$.)

(r1). For $t, u=1, \ldots, q,\left(\partial / \partial \beta_{t}\right) f(x ; \boldsymbol{\beta})$ and $\left(\partial^{2} / \partial \beta_{t} \partial \beta_{u}\right) f(x ; \boldsymbol{\beta})$ exist almost everywhere and are such that for each $\boldsymbol{\beta}_{0} \in B_{0}$ uniformly in a neighborhood of $\boldsymbol{\beta}_{0}$

$$
\left|\left(\partial / \partial \beta_{t}\right) f(x ; \boldsymbol{\beta})\right| \leq H_{t}(x)
$$

and

$$
\left|\left(\partial^{2} / \partial \beta_{t} \partial \beta_{u}\right) f(x ; \boldsymbol{\beta})\right| \leq G_{t u}(x),
$$

where

$$
\int_{\mathbb{R}} H_{t}(x) d x<\infty
$$

and

$$
\int_{\mathbb{R}} G_{t u}(x) d x<\infty:
$$

(r2). For $t, u=1, \ldots, q,\left(\partial / \partial \beta_{t}\right) \log f(x ; \boldsymbol{\beta})$ and $\left(\partial^{2} / \partial \beta_{t} \partial \beta_{u}\right)$ $\log f(x ; \boldsymbol{\beta})$ exist almost everywhere and are such that the Fisher information matrix,

$$
\mathbf{I}_{\boldsymbol{\beta} \boldsymbol{\beta}}=E_{\boldsymbol{\beta}}\left\{\left[\frac{\partial}{\partial \boldsymbol{\beta}} \log f\left(X_{1} ; \boldsymbol{\beta}\right)\right]\left[\frac{\partial}{\partial \boldsymbol{\beta}} \log f\left(X_{1} ; \boldsymbol{\beta}\right)\right]^{\prime}\right\},
$$

is finite, positive definite, and continuous and, as $\delta \rightarrow 0$, we have

$$
\begin{aligned}
E_{\boldsymbol{\beta}}\left\{\sup _{\{\mathbf{h}:\|\mathbf{h}\| \leq \delta\}} \| \frac{\partial^{2}}{\partial \boldsymbol{\beta} \partial \boldsymbol{\beta}^{\prime}} \log f\left(X_{1} ; \boldsymbol{\beta}+\mathbf{h}\right)\right. \\
\left.\quad-\frac{\partial^{2}}{\partial \boldsymbol{\beta} \partial \boldsymbol{\beta}^{\prime}} \log f\left(X_{1} ; \boldsymbol{\beta}\right) \|\right\} \rightarrow 0 .
\end{aligned}
$$

(r3). For each $\boldsymbol{\beta}_{0} \in B_{0}$, there exists $\eta=\eta\left(\boldsymbol{\beta}_{0}\right)>0$ with

$$
\sup _{\left\|\boldsymbol{\beta}-\boldsymbol{\beta}_{0}\right\|<\eta} \sup _{x \in \mathbb{R}}\left|\frac{\partial^{2}}{\partial \beta_{t} \partial \beta_{u}} F(x ; \boldsymbol{\beta})\right|<\infty \quad t, u=1, \ldots, q
$$

and

$$
\sup _{x \in \mathbb{R}}\left|\frac{\partial}{\partial \beta_{t}} F(x ; \boldsymbol{\beta})\right|_{\boldsymbol{\beta}=\boldsymbol{\beta}_{0}}<\infty \quad t=1, \ldots, q .
$$

(r4). There exist positive constants $c_{1}, c_{2}, \rho_{1}$, and $n_{1}$ such that

$$
P_{\boldsymbol{\beta}}(\sqrt{n}\|\hat{\boldsymbol{\beta}}-\boldsymbol{\beta}\| \geq r) \leq c_{1} \exp \left(-c_{2} r^{2}\right)
$$

for all $r=\rho \sqrt{\log n}$ with $0<\rho \leq \rho_{1}$ and $n \geq n_{1}$.

(r5). There exists $\boldsymbol{\beta} \in B$ such that

$$
\|\hat{\boldsymbol{\beta}}-\boldsymbol{\beta}\| \rightarrow 0
$$

under the alternative $P$.

(r6). For $\beta$ from (r5), there exists $\eta>0$ such that

$$
\sup _{\|\boldsymbol{\gamma}-\boldsymbol{\beta}\|<\eta} \sup _{x \in \mathbb{R}}\left|\frac{\partial}{\partial \gamma_{t}} F(x ; \gamma)\right|<\infty \quad t=1, \ldots, q .
$$

The next conditions concern the orthonormal system.

(s1). $\sup _{x \in[0,1]}\left|\phi_{j}^{\prime}(x)\right| \leq c_{3} j^{m_{1}}$ for each $j=1,2, \ldots, d(n)$ and some $c_{3}>0, m_{1}>0$.

(s2). $\sup _{x \in[0,1]}\left|\phi_{j}^{\prime \prime}(x)\right| \leq c_{4} j^{m_{2}}$ for each $j=1,2, \ldots, d(n)$ and some $c_{4}>0, m_{2}>0$.

Finally, we have conditions on the dimension $d(n)$ of the exponential family:

(d1). $\left\{d(n) V_{d(n)}\right\}^{2} n^{-1} \log n \rightarrow 0$ as $n \rightarrow \infty$, where $V_{k}=$ $\max _{1 \leq \jmath \leq k} \sup _{x \in[0,1]}\left|\phi_{\jmath}(x)\right|$.

(d2). $d(n)=o\left(\{n / \log n\}^{(2 m)^{-1}}\right)$ as $n \rightarrow \infty$, where $m=$ $\max \left(m_{1}, m_{2}\right)$.

(d3). $d(n)=o\left(n^{c}\right)$ as $n \rightarrow \infty$ for some $c<c_{2} b^{-2}$ if $\rho_{1} b \geq 1$, and with $c=c_{2} \rho_{1}^{2}$ otherwise, where $c_{2}$ and $\rho_{1}$ are given by (r4) and

$$
b=\left\{\sum_{t=1}^{q} \operatorname{var}_{\boldsymbol{\beta}} \frac{\partial}{\partial \beta_{t}} \log f(X ; \boldsymbol{\beta})\right\}^{1 / 2} .
$$

\section{Proof of Corollary 2.1}

Conditions (r1)-(r3) and (r6) can be easily checked by direct calculations. Condition ( $\mathrm{r} 5$ ) follows from the law of large numbers. Condition (r4) is obtained by application of standard moderate deviation theory. Both in the exponential case and in the normal case, it holds with $c_{2}=1 / 2\left(\operatorname{var}_{\beta} X\right)^{-1}$, whereas $\rho_{1}$ may be taken as large as one wants. We omit the details (see, e.g. Kallenberg 1983 ex. 2.1 , p. 502). Hence (d3) reads as $d(n)=o\left(n^{c}\right)$ for some $c<1 / 2$ in the exponential case and as $d(n)=o\left(n^{c}\right)$ for some $c<1 / 6$ in the normal case. Therefore, (d3) is satisfied under the conditions on $d(n)$ stated in the corollary.

Further, for the orthonormal Legendre polynomials on $[0,1]$ (s1) and (s2) are fulfilled with (Sansone 1959, p. 251) $m_{1}=5 / 2$ and $m_{2}=9 / 2$. For the cosine system, (s1) and (s2) are fulfilled with $m_{1}=1$ and $m_{2}=2$.

Because for the orthonormal Legendre polynomials on $[0,1]$, we get $V_{k}=(2 k+1)^{1 / 2}$ (Sansone 1959, p. 190), (d1) reduces in this case to $\{d(n)\}^{3} n^{-1} \log n \rightarrow 0$ as $n \rightarrow \infty$, and hence (d1) is satisfied. For the cosine system, we have $V_{k}=\sqrt{2}$ and hence 
(d1) reduces in this case to $\{d(n)\}^{2} n^{-1} \log n \rightarrow 0$ as $n \rightarrow \infty$, implying that also for the cosine system, (d1) is satisfied. Finally, (d2) exactly equals the condition on $d(n)$ in the corollary.

Because all conditions of Theorem 2.1 are satisfied, the theorem may be applied, thus completing the proof of Corollary 2.1 .

[Received March 1995. Revised December 1996.]

\section{REFERENCES}

Ascher, S. (1990), "A Survey of Tests for Exponentiality," Communications in Statistics, Part A-Theory and Methods, 19, 1811-1825.

Bickel, P. J., and Doksum, K. A. (1977), Mathematical Statistics, Basic Ideas and Selected Topics, San Francisco: Holden-Day.

Bickel, P. J., and Ritov, Y. (1992), "Testing for Goodness of Fit: A New Approach," in Nonparametric Statistics and Related Topics, ed. A.K.Md.E. Saleh, Amsterdam: North-Holland, pp. 51-57.

Bogdan, M. (1995), "Data-Driven Versions of Pearson's Chi-Square Test for Uniformity," Journal of Statistical Computation and Simulation, 52, 217-237.

Eubank, R. L., and Hart, J. D. (1992), "Testing Goodness-of-Fit in Regression via Order Selection Criteria," The Annals of Statistics, 20, 14121425.

Eubank, R. L., Hart, J. D., and LaRiccia, V. N. (1993), "Testing Goodness of Fit via Nonparametric Function Estimation Techniques," Communications in Statistics, Part A-Theory and Methods, 22, 3327-3354.

Eubank, R. L., and LaRiccia, V. N. (1992), "Asymptotic Comparison of Cramér-von Mises and Nonparametric Function Estimation Techniques for Testing Goodness-of-Fit," The Annals of Statistics, 20, 2071-2086.

Fan, J. (1996), "Tests of Significance Based on Wavelet Thresholding and Neyman's Truncation," Journal of the American Statistical Association, 91, 674-688.

Gail, M. H., and Gastwirth, J. L. (1978), "A Scale-Free Goodness-of-Fit Test for the Exponential Distribution Based on the Gini Statistic," Journal of the Royal Statistical Society, Ser. B, 40, 350-357.

Gan, F. F., and Koehler, K. J. (1990), "Goodness-of-Fit Tests Based on P-P Probability Plots," Technometrics, 32, 289-303.

Hannan, E. J., and Quinn, B. G. (1979), "The Determination of the Order of an Autoregression," Journal of the Royal Statistical Society, Ser. B, 41, 190-195.

Haughton, D., Haughton, J., and Izenman, A. (1990), "Information Criteria and Harmonic Models in Time Series Analysis," Journal of Statistical Computation and Simulation, 35, 187-207.

Inglot, T., Kallenberg, W. C. M., and Ledwina, T. (1994a), "Power Approximations to and Power Comparison of Certain Goodness-of-Fit Tests," Scandinavian Journal of Statistics, 21, 131-145.

(1994b), "On Selection Rules With an Application to Goodness-
of-Fit for Composite Hypotheses," Memorandum 1242, University of Twente, Faculty of Applied Mathematics.

- (in press), "Data-Driven Smooth Tests for Composite Hypotheses," submitted to The Annals of Statistics, 25.

Inglot, T., and Ledwina, T. (1996), "Asymptotic Optimality of Data-Driven Neyman's Tests for Uniformity," The Annals of Statistics, 24, 19822019.

Javitz, H. S. (1975), "Generalized Smooth Tests of Goodness of Fit, Independence, and Equality of Distributions," unpublished Ph.D. dissertation, University of California, Berkeley, Dept. of Statistics.

Kallenberg, W. C. M. (1983), "On Moderate Deviation Theory in Estimation," The Annals of Statistics, 11, 498-504.

Kallenberg, W. C. M., and Ledwina, T. (1994), "Data-Driven Smooth Tests for Composite Hypotheses," Memorandum 1232, University of Twente, Faculty of Applied Mathematics.

(1995a), "Consistency and Monte Carlo Simulation of a DataDriven Version of Smooth Goodness-of-fit Tests," The Annals of Statistics, 23, 1594-1608.

(1995b), "On Data-Driven Neyman's Tests," Probability and Mathematical Statistics, 15, 409-426.

Kopecky, K. J., and Pierce, D. A. (1979), "Efficiency of Smooth Goodnessof-Fit Tests," Journal of the American Statistical Association, 74, 393397.

LaRiccia, V. N. (1991), "Smooth Goodness-of-Fit Tests: A Quantile Function Approach," Journal of the American Statistical Association, 86, 427-431.

Ledwina, T. (1994), "Data-Driven Version of Neyman's Smooth Test of Fit," Journal of the American Statistical Association, 89, 1000-1005.

Milbrodt, H., and Strasser, H. (1990), "On the Asymptotic Power of the Two-Sided Kolmogorov-Smirnov Test," Journal of Statistical Planning and Inference, 26, 1-23.

Neyman, J. (1980), "Some Memorable Incidents in Probabilistic/Statistical Studies," in Asymptotic Theory of Statistical Tests and Estimation, ed. I. M. Chakravarti, New York: Academic Press, pp. 1-32.

Rayner, J. C. W., and Best, D. J. (1989), Smooth Tests of Goodness of Fit, New York: Oxford University Press.

(1990), "Smooth Tests of Goodness of Fit: An Overview," International Statistical Review, 58, 9-17.

Risebrough, R. W. (1972), "Effects of Environmental Pollutants Upon Animals Other Than Man," in Proceedings of the Sixth Berkeley Symposium on Mathematical Statistics and Probability, Vol. VI, Berkeley: University of California Press, pp. 443-463.

Sansone, G. (1959), Orthogonal Functions, New York: Interscience.

Schwarz, G. (1978), "Estimating the Dimension of a Model," The Annals of Statistics, 6, 461-464.

Thomas, D. R., and Pierce, D. A. (1979), "Neyman's Smooth Goodness-ofFit Test When the Hypothesis is Composite," Journal of the American Statistical Association, 74, 441-445. 